\title{
Blood substitutes- the polyheme trials
}

\author{
Sameer S. Apte*
}

\section{PROLOGUE: A HYPOTHETICAL CASE}

A 35 year old female, victim of a motor vehicle accident, sustained life threatening injuries leading to hemorrhagic shock. When paramedics arrived at the scene the patient was unconscious. Due to acute blood loss, the patient's blood pressure and oxygen saturation were critically low, leaving only a short therapeutic window to save the patient. The paramedics were unsure whether the current standard of care, an infusion of saline, would be adequate to sustain the patient's life until the nearest hospital could be reached. These paramedics, however, have been instructed to enroll hemorrhagic shock victims in a phase III clinical trial to test the efficacy and safety of an artificial oxygen carrying fluid or "blood substitute". Like saline, the blood substitute would increase the patient's blood pressure. In addition, it could also help supply tissues with much needed oxygen, potentially reducing the chance of mortality.

Because the patient is unconscious, however, the paramedics are placed at the center of an ethical dilemma. If the injuries are sufficiently severe, infusion of the experimental fluid may maintain the patient's vitals long enough to be safely transported to a trauma center. The patient, however, is incapacitated, so there is no feasible way to obtain proper informed consent.

Polyheme ${ }^{\circledR}$, a blood substitute, has completed a pivotal phase III clinical trial in which the ethical dilemma exemplified in this hypothetical situation was commonplace (1). Because this clinical trial examines a new emergency therapy, the Food and Drug Administration (FDA) invoked Title 21, Section 50.24, Subpart B of the Code of Federal Regulations in order to grant the creators of Polyheme, Northfield laboratories Inc. (Illinois, USA), an exemption from acquiring informed consent (2-5).

The waiver of informed consent has caused the clinical trial itself to become the center of an intense

*To whom correspondence should be addressed:

Sameer S. Apte

McGill University, QC, CAN

Email: sameer.apte@mail.mcgill.ca ethical debate (1, 6-15). Opponents say that Northfield Laboratories has inappropriately influenced regulatory decisions and abused the bureaucratic system in order to gain approval for a questionable experimental protocol $(7,10,13)$. Conversely, proponents argue that prolonged suspension of informed consent is a necessity for effective characterization of safety and efficacy of prospective emergency therapies $(6,12,14)$. This putative necessity is hinged upon the fact that the majority of patients targeted by new emergency therapies are incapacitated at the time of enrollment (2$4,16)$. What is not debated is that the completion of the pivotal phase III clinical trial for Polyheme is surrounded by a cloud of ethical tension...

\section{THE ORIGIN OF BLOOD TRANSFUSIONS \& ARTIFICIAL BLOOD}

In 1909, the successful characterization of the "ABO" and "Rhesus" blood type antigens on the surface of the red blood cell (RBC) allowed clinicians to begin transfusing trauma patients with whole allogeneic blood $(17,18)$. Since then, allogeneic blood transfusions have become ubiquitous in clinical medicine $(19,20)$. Interestingly, even though blood transfusions are the gold standard of care, the efficacy and safety of allogeneic red cell therapy has never been rigorously tested via the clinical trial process (20-22). Indeed, the indications for treatment are largely based on common medical practice, tradition, and expert advice (20-22). Thus, comparing the safety and efficacy of a blood substitute to the standard of care may prove to be difficult.

The most serious motivation for the development of a blood substitute is the worldwide shortage of safe and viable allogeneic donor blood (7). A recent report on blood donations found that during 2001, $12.7 \%$ of hospitals reported a cancellation of surgeries due to donor blood shortage and $18.9 \%$ reported a shortage of blood for non-surgical purposes (19). In addition, the stress on the donated blood supply is projected to increase in the coming years (23).

In spite of many research initiatives, blood substitute investigations are not yet focused on providing a long 
term alternative for allogeneic whole blood. The acute conditions currently targeted by blood substitutes include hemorrhagic shock or ischemic stroke $(24,25)$. Because these pathophysiologies are encountered primarily in emergency situations $(26,27)$, clinical applications of blood substitutes aim only to prolong patient survival until allogeneic blood transfusions become available (27-30).

Outside of the hospital, long-term storage of allogeneic blood is impractical and donor blood type cannot be cross-matched to the recipient (7). Thus, paramedics are restricted to saline, a volume expander, as the standard treatment for hemorrhagic shock (11, 12). Like saline, blood substitutes replenish lost blood volume, do not carry blood type antigens, and are stable for long periods of time at room temperature (31-33). In addition, blood substitutes can facilitate oxygen delivery to ischemic tissues, highlighting an important advantage over the use of saline(34).

In the hospital, in spite of enhanced screening methods for blood-borne illnesses, it is still impossible to be certain that allogeneic transfusions are free of pathogens $(6,30,35)$. For example, during the remission periods of retroviral life cycles, viral particles cannot be detected (36). Many serious infections including HIV, hepatitis B \& C, malaria, vCJD and cytomegalovirus are still transmitted by transfusions $(20,36)$. Blood substitutes avoid this risk by undergoing pasteurization, ultrafiltration or other sterilization techniques (32, 36-39).

Lastly, a potential market niche for blood substitutes is the population of patients that refuse allogeneic blood transfusion due to religious beliefs $(34,40)$. For a blood substitute to be suitable for those with special religious needs, no part can be derived from an animal or human source $(21,28)$. Compassionate use of Polyheme has already been clinically implemented in cases where allogeneic blood transfusions are refused (41).

The potential for blood substitutes to address the problems with current therapies has been recognized. To date, investigation of blood substitutes has fostered over eighteen clinical trials resulting in granted licensures in multiple foreign countries $(26,27,33,37$, $42,43)$.

\section{AN OVERVIEW OF HEMOGLOBIN-BASED OXYGEN CARRIERS}

The founder of the field of blood substitutes is Dr. Thomas Chang of McGill University, who in the late 1950s, encapsulated hemoglobin molecules in a polyvinyl chloride (PVC) membrane (44). Since then, two major categories of blood substitutes have arisen: perfluorocarbons (PFCs) and hemoglobin-based oxygen carriers (HBOCs). PFCs are chemically inert organic fluids with the ability to dissolve 20 times as much oxygen as plasma $(38,39,45)$. HBOCs, like Polyheme, are solutions of modified hemoglobin in plasma-like fluids. In addition to a reduced side-effect profile, HBOCs deliver oxygen to tissues through facilitated diffusion (via hemoglobin) instead of passive diffusion $(27,46)$. Thus, HBOCs have proven more efficacious than PFCs in clinical settings $(34,47)$.

Because extracellular hemoglobin degrades into nephrotoxic dimers, initial attempts to use free-floating or "stroma-free" hemoglobin (SFHb) have failed (46). In addition, because hemoglobin extravasates into endothelial cells and binds the vasodilator nitric oxide, it is a vasoconstrictor (48). To alleviate these problems, various methods to modify hemoglobin including crosslinking, conjugation, and polymerization have been investigated(33).

The earliest endeavours to prevent the dimerization of $\mathrm{SFHb}$ used cross-linking agents like diaspirin or glutaraldehyde to covalently bind hemoglobin's subunits together $(49,50)$. While nephrotoxicity was reduced somewhat, because cross-linked SFHb is still small enough to extravasate into endothelial cells, its vasoactive properties were still significant $(48,50)$. Furthermore, cross-linked hemoglobin caused cardiac toxicity (27).

In addition to reducing nephrotoxicity, increasing the molecular weight of SFHb by conjugation with polyethylene glycol or malemide has been shown to lengthen intra-vascular half-life, thereby reducing vasoactivity (51). One pre-market conjugated $\mathrm{SFHb}$ solution, Hemospan, has shown moderate efficacy and an acceptable safety profile after phase II clinical trials (47). The manufacturers, Sangart Inc. (California, USA), will soon begin phase III clinical trials (27).

The last common modification of SFHb polymerizes tetramers into macromolecular chains (28). This process affords the same benefits as cross-linking and conjugation $(31,34,50)$. By controlling the size of the polymers, oxygen disassociation characteristics and nitric oxide binding properties can also be fine tuned (29, 31, 50). Similarly to Northfield Laboratories' Polyheme, Biopure Inc. (Massachusetts, USA) has manufactured a polymerized bovine hemoglobin formulation called Hemopure (29). Bovine SFHb comes from a plentiful and renewable resource and has the advantage of not requiring 2,3-diphosphoglycerate to facilitate proper oxygen offloading $(27,33)$. Hemopure is currently licensed in South Africa and pivotal phase III trials have been completed in North America for use in orthopaedic surgery (46).

Northfield Laboratories' Polyheme is an HBOC synthesized by polymerization of hemoglobin extracted from expired human donor blood (34). Due to 
purification and polymerization, an appreciable vasoconstrictory effect is not associated with Polyheme and no adverse cardiovascular events were reported in preliminary safety studies $(31,34)$. Recently, Polyheme has completed a controversial pivotal phase III clinical trial and may be the first HBOC to enter Phase IV marketing trials $(27,33,37)$.

\section{THE POLYHEME CLINICAL TRIALS: ETHICS AND EDITORIAL}

In 1996, the Department for Health and Human Services (DHHS) and the FDA enacted complementary regulations for waiving the requirement for informed consent in certain emergency research protocols (2-4). 21CFR50.24 and 45CFR46 are federal regulations called the 'Emergency Research Waiver' (ERW) rules that allow an incapacitated person to be enrolled in a clinical trial without consent of a legally authorized representative, provided that the following criteria are fulfilled $(2-4,10)$ :

1. The subject must be suffering from a lifethreatening condition necessitating immediate treatment.

2. Obtaining informed consent from a legally authorized representative must be infeasible given the time frame in which the subject must be treated.

3. Previous clinical evidence must indicate that the investigated therapy will grant a serious benefit to the subject.

4. The investigators must make an effort to contact a legally authorized representative during the given therapeutic window.

5. An independent committee must review collected data of the ongoing study.

6. Available treatments must be "unproven and unsatisfactory" (2).

7. Principal investigators must meet with the communities from which the subjects will be drawn, and publicly disclose pertinent aspects of the study including the risks and benefits.

Potentially life-saving therapies for which there are no other methods of treatment can also be given special consideration under federal law. A "Special Protocol Assessment" (SPA) is a stipulation outlined in the 1997 US Modernization Act $(52,53)$ that allows a clinical trial to be streamlined for fast approval; trials which led to the installation of publicly available defibrillators, for instance, were conducted under this stipulation (54). To be eligible, the experimental therapy must have no existing alternative. The FDA has deemed that Polyheme meets these requirements and has granted
Northfield Laboratories SPA status for Polyheme (10, $55)$.

The FDA and DHHS regulations attempt to find a balance between expediting approval for emergency therapies and ethical practices of clinical investigation. In some cases, however, the interpretation of these regulations can lead to questionable practices.

Having commenced phase I clinical trials in 1991, Polyheme's journey through pivotal phase III studies just recently finished in May 2007 (56, 57). Early trials to determine the efficacy and safety of large volume Polyheme infusions (up to 20 units) were completed in 2002 (56, 58). These secondary studies showed Polyheme's capability to sustain patient survivability in conditions of extreme endogenous hemoglobin deprivation, where death is statistically certain $(31,34)$. After reviewing the results, the FDA granted Polyheme permission to sponsor a pivotal phase III clinical trial involving 720 hemorrhagic shock victims, pursuant under the ERW $(11,13,57)$.

The phase III clinical trial was conducted in conjunction with 32 trauma centers across 19 US states and continued from July 2006 to May $2007(5,57)$. The trial has two distinct phases, a "pre-hospital" phase, and an "in-hospital" phase. In the pre-hospital phase, incapacitated trauma patients are randomized between Polyheme and the current standard of care, saline. Upon arrival at the hospital, patients previously given saline in the pre-hospital phase are given allogeneic blood, while patients previously given Polyheme in the prehospital phase are continued on the Polyheme treatment for up to 12 hours. The in-hospital phase of this study uses Polyheme in spite of the availability of allogeneic whole blood (5). Furthermore, if the patient was enrolled in the trial while unable to give informed consent, infusion of Polyheme at the hospital site can be performed without proper notification either the subjects themselves or a legally authorized representative $(3,10,11)$.

The pre-hospital phase of this trial is not heavily debated and the necessity of the ERW rule is widely accepted (6-14). The in-hospital phase of this study, however, is heavily debated. The major point of contention comes from the fact that patients who were randomized to the Polyheme group in the pre-hospital phase were not treated with allogeneic whole blood transfusions upon arrival at the hospital (6-14). Previous studies performed under the ERW, most notably the public access defibrillator trial, did not have a protocol with a questionable in-hospital phase $(54,59,60)$. Thus, the ethics pertaining to informed consent in the Polyheme clinical trial present a novel situation.

Opponents of the in-hospital phase do not discount the scientific validity of a clinical comparison between 
Polyheme and allogeneic whole blood, but they charge that such a comparison must be conducted with the full cooperation and informed consent of patients or power of attorney $(10,13)$. A further point of contention exists due to dysfunction in the regulatory mechanisms for clinical trials falling under the ERW and the SPA. In particular, in 2006 Kipnis et al. uncovered a number of hindrances to the effectiveness of institutional review board (IRB) and FDA consideration of the Polyheme trial (10).

First, Northfield Laboratories was allowed to maintain secrecy of the specifics of the experimental protocol and research methodologies relevant to the study. Only after signing non-disclosure forms were IRBs and the FDA allowed to review the study protocol (10). In addition, although Northfield Laboratories did discuss aspects of the study including the risks and benefits, the study protocol itself was not disclosed at community meetings; there is no requirement to do so under the ERW $(2,4,61)$. It is reasonable that a company should be permitted to protect its intellectual property; the nondisclosure, however, of the Polyheme research protocol seems to be in direct contradiction with stipulations six and seven as previously outlined in the criteria to be granted an ERW $(2,4)$. In the case of an ERW, community consultations are essentially a surrogate method to facilitate informed consent $(3,10,15)$. For all intents and purposes, ERW clinical trials enroll an entire community as test subjects. Accordingly, the requirements for surrogate informed consent should be more stringent than for studies where only one subject is being implicated. For example, in a more recent ERW clinical trial that tests the "ResQPump", an automated CPR device, the investigators went to great lengths to fulfill the requirement for community consultations. Specifically, 136 community organizations were contacted, major television and radio networks were sent press releases, 2 local newspapers were informed and multiple public service announcements were aired on television and radio. Because of this effort, attendance at community consultation meetings was tripled over previous ERW Clinical trials (59).

A second obstacle arises because the process by which an IRB reviews a clinical trial protocol is hindered by ineffective regulatory mechanisms and the interplay between ERA and SPA conditions $(3,10,11)$. Normally, when an IRB decides to disapprove of a multi-center study protocol, it is required to report their rejection to the sponsor of the study, the local investigator, the FDA, and all other IRBs reviewing the same study or studies with similar protocols $(2,4)$.

In this case, however, the research of Kipnis et al. revealed two mechanisms utilized by Northfield Laboratories to prevent IRBs from reporting their concerns. Before one IRB evaluation, the principal investigator of the Polyheme trial was privy to information that one member of the IRB had reservations about the in-hospital phase of the trial. Before the IRB convened to deliberate about the study, the application for approval of the trial at that site was withdrawn by Northfield Laboratories (10). In another case, one IRB returned the application for approval to Northfield Laboratories citing concerns pertaining to the in-hospital phase of the study. In order to allow Northfield Laboratories a chance to address these concerns, the IRB requested that they amend and resubmit their request for ERW approval. Northfield Laboratories simply elected not to respond and to pursue study opportunities in other cities. Because the IRB never formally denied the study, a report was never authored, and the FDA and other IRBs were not informed $(10,11)$.

In the first case, a lack of confidentiality pertaining to IRB proceedings allowed Northfield Laboratories to avoid regulatory mechanisms in order to prevent an unfavourable outcome. In the second instance, an unwarranted decision by an IRB allowed Northfield Laboratories to sidestep regulatory mechanisms. In order to deter clinical trial sponsors from attempting to bypass regulatory procedures, two rules should be enacted:

1. Once a protocol has been submitted for assessment, the sponsor may not retract the submission.

2. Irrespective of whether a sponsor intends to continue pursuing a clinical trial for a submitted protocol, the IRB should evaluate it and officially inform relevant organizations of their findings.

Implementing these two regulations creates an incentive for sponsors to be absolutely certain the submitted protocol is of the highest integrity.

Another notable obstacle to effective review of the Polyheme protocol is the lack of malleability in the regulatory elements of the SPA. The SPA specifies that once the FDA and sponsor confirm an SPA agreement, the study protocol is locked until completion of the study. Furthermore, SPA status can only be granted before the study is evaluated. There are only four allowable exceptions to these rules $(52,53)$ :

1. A mutual written agreement is formulated between sponsor and FDA outlining changes to be made.

2. A "substantial scientific issue essential to determining the safety or effectiveness of the drug" is characterized as unacceptable.

3. The sponsor does not adhere to the agreed upon 
study protocol.

4. "The relevant data, assumptions, or information provided by the sponsor in a request for special protocol assessment change are found to be false statements, misstatements or are found to omit relevant facts."

If a sponsor wishes to change a protocol, a new SPA must be applied for $(52,53)$. Additionally, the ERW implies that protocols should be modified in response to community concerns $(2,4)$. Taken together, these facts suggest that sponsors may dissuaded from being candid towards community consultations in order to prevent possible reapplication for an SPA to modify the study protocol.

It should also be noted that the four exceptions for change of an SPA do not include any mention of ethical concerns voiced either by IRBs or by the community $(52,62)$. Because of this, the degree to which the FDA can alter an SPA due to ethical concerns is unknown. Thus, it is unclear whether the concerns voiced about the in-hospital phase of the Polyheme trial could have been legally addressed by the FDA.

In May 2007, Northfield Laboratories released preliminary results of the pivotal phase III clinical trial of Polyheme and have applied for a marketing license from the FDA (57). In spite of the concerns revealed by the research of bioethicists such as Kipnis et al., at the current time, the FDA has not made a public statement regarding the protocol of the Polyheme trials. Furthermore, the ambiguities in the SPA and ERW conditions have not been formally addressed and the regulations remain as is $(2,4,52,53)$.

\section{CONCLUSIONS}

The conflict in policy between the SPA guidelines and the ERW creates uncertainty in the regulatory process. The ambiguity of the guidelines for IRB evaluation leaves ample room for clinical trial sponsors to avoid unfavourable outcomes. More tightly regulating the submission and review process could alleviate this problem.

In light of the safety and efficacy demonstrated in pre-phase III trials of Polyheme, the potential clinical benefits to incapacitated subjects by participation in the Polyheme clinical trial clearly outweigh the potential risks. For this reason, it is imperative that regulatory statutes such as the ERW and SPA exist to facilitate speedy investigation of potentially lifesaving therapies like Polyheme. Once, however, an incapacitated subject enters a situation in which a satisfactory treatment is available, he possesses an inherent right to be given the standard treatment. The absence of a requirement in the Polyheme clinical trial to administer allogeneic blood to victims upon arrival at the hospital is unethical. Thus, the regulations concerning the informed consent of incapacitated patients for clinically investigating emergency therapies should be reviewed.

In spite of the concerns surrounding the design of the Phase III clinical trials, Polyheme needed to be given the opportunity to prove its efficacy in a clinical setting. If Polyheme fulfills the promises its creators have made, clinical outcomes for pre-hospital hemorrhagic shock victims will be drastically improved.

\author{
APPENDIX - ABBREVIATIONS \\ RBC - Red Blood Cell \\ HBOC - Hemoglobin Based Oxygen Carrier \\ $\mathrm{SFHb}$ - Stroma-Free Hemoglobin \\ PFC - Perfluorochemical \\ HIV - Human Immunodeficiency Virus \\ vCJD - Variant Creutzfeldt-Jakob Disease \\ IRB - Institutional Review Board \\ FDA - Food and Drug Administration \\ DHHS - Department of Health and Human Services \\ ERW - Emergency Research Waiver \\ SPA - Special Protocol Assessment
}

\section{REFERENCES}

1. Dickert, N.W. and J. Sugarman, Getting the ethics right regarding research in the emergency setting: lessons from the PolyHeme study. Kennedy Inst Ethics J, 2007. 17(2): p. 153-69.

2. Comm-Health, 21CFR50.24 - Informed Consent of Human Subjects, FDA, Editor. 1996, Health and Human Resources.

3. Biros, M.H., Research without Consent: Exceptin from and Waiver of Informed Consent in Resuscitation Research. Sci Eng Ethics, 2007. Epub ahead of Print.

4. Ellis, G.B. and M.H. Lin. (45CFR Part 46) OPRR Reports: Informed Consent Requirements in Emergency Research. 1996 (cited 97-01; Available from:

http://www.hhs.gov/ohrp/humansubjects/guidance/hsdc9701.htm.

5. Northfield-Laboratories. Safety and Efficacy of PolyHeme(R) in Hemorrhagic Shock Following Traumatic Injuries Beginning in the Pre-Hospital Setting. 2004 July 31st 2006 (cited 2008 January 15th); Available from: http://clinicaltrials.gov/ct2/show/NCT00076648.

6. Dougherty, A.H., Letter to the Editor: In Defense of the PolyHeme Trial. Am J Bioeth, 2006. 6(5): p. W35.

7. Holloway, K.F.C., Accidental Communities: Race, Emergency Medicine, and The Problem of PolyHeme. Am J Bioeth, 2006. 6(3): p. 1-11.

8. Holloway, K.F.C., Response to Open Peer Commentaries on "Accidental Communities: Race, Emergency Medicine, and the Problem of PolyHeme": The "R" Word: Bioethics and a (Dis)Regard of Race. Am J Bioeth, 2006. 6(3): p. W46-W48.

9. Kipnis, K., N.M.P. King, and R.M. Nelson, Response to Open Peer Commentaries on "An Open Letter to Institutional Review Boards Considering Northfield Laboratories' PolyHeme ${ }^{\circledR}$ Trial": The Emergency Exception and Unproven/Unsatisfactory Treatment. Am J Bioeth, 2006. 6(3): p. W49-W50.

10. Kipnis, K., N.M.P. King, and R.M. Nelson, Trials and Errors: Barriers to Oversight of Research Conducted Under the 
Emergency Research Consent Waiver. IRB - The Hastings Center, 2006. 28(2): p. 16-19.

11. Kipnis, K., N.M.P. King, and R.M. Nelson, An Open Letter to Institutional Review Boards Considering Northfield Laboratories' PolyHeme ${ }^{\circledR}$ Trial. Am J Bioeth, 2006. 6(3): p. 1821.

12. McKenna, M., Polyheme and the Ethics of Informed Consent Ann Emerg Med, 2006. 48(5): p. 563-566.

13. Shamoo, A.E., Letter to the Editor: Emergency Research Consent Waiver - A Proper Way. Am J Bioeth, 2006. 6(4): p. W48.

14. Molter, N.C., Exemption of informed consent (final rule): procedures for critical trauma studies. J Trauma, 2007. 62(6 Suppl): p. S78-9.

15. Longfield, J.N., et al., Community meetings for emergency research community consultation*. Crit Care Med, 2007.

16. Moore, K.M., Controversies in Fluid Resuscitation. J Trauma Nurs, 2006. 13(4): p. 168-172.

17. Landsteiner, Karl Landsteiner - Biography. Nobel Lectures Physiology or Medicine 1922-1941. 1965, Amsterdam: Elsevier Publishing Company.

18. Spiess, B.D., Choose one: Damned if you do/damned if you don't! Crit Care Med, 2005. 33(8): p. 1871-1874.

19. Sullivan, M.T., et al., Blood collection and transfusion in the United States in 2001. Transfusion, 2007. 47: p. 385-394.

20. Spiess, B.D., Risks of Transfusions: Outcome Focus. Transfusion, 2004. 4: p. 4S-14S.

21. Sazama, K., The Ethics of Blood Management. Vox Sanguinis, 2007. 92: p. 95-102.

22. Cable, R., et al., Practice Guidelines for Blood Transfusion: A Compilation from Recent Peer Reviewed Literature. 2002, Washington: American National Red Cross.

23. Zou, S., et al., Changing age distribution of the blood donor population in the United States. Transfusion, 2007.

24. Spiess, B.D., The Body, in Discovery 2057. 2007, Discovery Channel, Inc.: United States. p. 60min.

25. Kim, H.W. and A.G. Greenburg, Toward 21st Century Blood Component Replacement Therapeutics: Artificial Oxygen Carriers, Platelet Substitutes, Recombinant Clotting Factors, and Others. Art Cells Blood Subs Immob Biotech, 2006. 34: p. 537-550.

26. Mosa, M.M. and D.C.H. Cheng, Oxygen Therapeutics (Blood Substitutes) in Cardiac Surgery. Curr Opin Anaesthesiol, 2003. 16: p. 21-26.

27. Winslow, R.M., Current status of oxygen carriers ('blood substitutes'). Vox Sanguinis, 2006. 91: p. 102-110.

28. Chang, T.M.S., Blood Substitutes: Principles, Methods, Products and Clinical Trials. Vol. I. 1997, Basel: Karger Landes Systems.

29. Chang, T.M.S., Blood substitutes based on nanobiotechnology. Trends in Biotechnology, 2006. 24(8): p. 372-377.

30. Feit, A. and R.N. Sladen, New Haemoglobin Substitutes. Curr Opin Anaesthesiol, 1999. 12(4): p. 473-479.

31. Gould, S.A., et al., The First Randomized Trial of Human Polymerized Hemoglobin as a Blood Substitute in Acute Trauma and Emergent Surgery. J Am Coll Surg, 1998. 187(2): p. 113-120.

32. Habib, F.A. and S.M. Cohn, Blood substitutes. Curr Opin Anaesthesiol, 2004. 17: p. 139-143.

33. Stollings, J.L. and L.J. Oyen, Oxygen Therapeutics: Oxygen Delivery Without Blood. Pharmacotherapy, 2006. 26(10): p. 1453-1464.

34. Gould, S.A., et al., The Life-Sustaining Capacity of Human Polymerized Hemoglobin when Red Cells Might Be Unavailable. J Am Coll Surg, 2002. 195(4): p. 445-452.

35. Petricciani, J.C. and J.S. Epstein, The Effect of the AIDS
Epidemic on the Safety of the Nation's Blood Supply. Pub Health Reports, 1988. 103(3): p. 236-241.

36. Madjdpour, C., V. Heindl, and D.R. Spahn, Risks, Benefits, Alternatives and Indications of Allogeneic Blood Transfusions. Minerva Anestesiol, 2006. 72: p. 283-298.

37. Moore, E.E., et al., Hemoglobin-Based Oxygen Carriers in Trauma Care: Scientific Rationale for the US Multicenter Prehosptial Trial. World J Surg, 2006. 30: p. 1247-1257.

38. Spahn, D.R. and D. Pasch, Physiological Properties of Blood Substitutes. News Physiol Sci, 2001. 16: p. 38-41.

39. Riess, J.G., Perfluorocarbon-based Oxygen Delivery. Art Cells Blood Subs Immob Biotech, 2006. 34: p. 567-580.

40. Rogers, D.M. and K.P. Crookston, The approach to the patient who refuses blood transfusion. Transfusion, 2006. 46: p. 14711477 .

41. Smith, S.E., et al., The administration of polymerized human hemoglobin (Pyridoxylated) to a Jehovah's Witness after submyeloablative stem cell transplantation complicated by delayed graft failure. Compr Ther, 2006. 32(3): p. 172-5.

42. Tsuchida, E., et al., Hemoglobin Vessicles as a Transfusion Alternative. Art Cells Blood Subs Immob Biotech, 2006. 34: p. 581-588.

43. Fleming, P., Perceptions in Transfusion Medicine: A Pilot Field Study on Risk and Ethics for Blood and Blood Substitutes. Art Cells Blood Subs Immob Biotech, 2007. 35: p. 149-156.

44. Picard, A., The Red Blood Cell Man. McGill News, 1996. 76(4): p. 22-25.

45. Riess, J.G., Understanding the Fundamentals of Perfluorocarbons and Perfluorocarbon Emulsions Relevant to In Vivo Oxygen Delivery. Art Cells Blood Subs Immob Biotech, 2005. 33: p. 47-63.

46. Jahr, J.S., V. Walker, and K. Manoochehri, Blood substitutes as pharmacotherapies in clinical practice. Curr Opin Anaesthesiol, 2007. 20(4): p. 325-30.

47. Olofsson, C., et al., A multicenter clinical study of the safety and activity of maleimide-polyethylene glycol-modified Hemoglobin (Hemospan) in patients undergoing major orthopedic surgery. Anesthesiology, 2006. 105(6): p. 1153-63.

48. Doherty, D.H., et al., Rate of Reaction with Nitric Oxide Determines the Hypertensive Effect of Cell-Free Hemoglobin. Nature Biotech, 1998. 16: p. 672-676.

49. Schubert, A., et al., Diaspirin-Crosslinked Hemoglobin Reduces Blood Transfusion in Noncardiac Surgery: A Multicenter, Randomized, Controlled, Double-Blinded Trial. Anesth Analg, 2003. 97: p. 323-332.

50. Yu, B., Z. Liu, and T.M.S. Chang, Polyhemoglobin with Different Percentage of Tetrameric Hemoglobin and Effects on Vasoactivity and Electrocardiogram. Art Cells Blood Subs Immob Biotech, 2006. 34: p. 159-173.

51. Kim, H.W. and A.G. Greenburg, Artificial Oxygen Carriers as Red Blood Cell Substitutes: A Selected Review and Current Status. Art Organs, 2004. 28(9): p. 813-828.

52. Anonymous. Special Protocol Assessment, Guidance. 2002 [cited; Available from: http://www.fda.gov/cder/guidance/3764fnl.pdf.

53. Congress, U.S., Modernization Act, U.H.o.R.a.U. Senate, Editor. 1997.

54. Hallstrom, A.P., J.P. Ornato, and M. Weisfeldt, Public Access Defibrillation Trial Investigators. Public-access defibrillation and survival after out-of-hospital cardiac arrest. . NEJM, 2004. 351(7): p. 637-646.

55. Anonymous, Human haemoglobin - Northfield. BioDrugs, 2003. 17(4): p. 296-8

56. Gould, S.A., et al., Clinical experience with human polymerized hemoglobin. Transfusion, 1993. 33: p. 60S

57. Gould, S.A. Results of Pivotal Phase III Clinical Trial. 2007 
May 23rd [cited January 16th 2007); Available from: http://phx.corporate-ir.net/phoenix.zhtml?c=91374\&p=irolnewsArticle \&ID $=1005951$ \&highlight $=$.

58. Gould, S.A., et al., Clinical Utility of Human Polymerized Hemoglobin as a Blood Substitute after Acute Trauma and Urgent Surgery. Trauma Injury Infection Crit Care, 1997. 43(2): p. 325-332.

59. Salzman, J.G., et al., Implementing emergency research requiring exception from informed consent, community consultation, and public disclosure. Ann Emerg Med, 2007. 50(4): p. 448-55, 455 e1-4.

60. Lewis, R.J. and N. Fost, Monitoring a Clinical Trial With Waiver of Informed Consent: Diaspirin Cross-Linked Hemoglobin for Emergency Treatment of Post-Traumatic Shock, in Data Monitoring in Clinical Trials. 2006, Springer New York: New York.

61. Fulda, G. Polyheme Trauma Trial - Community Consultation. [Powerpoint presentation] 2006 May 15th [cited; 1-6]. Available from: http://www.fda.gov/ohrms/dockets/dockets/95s0158/95s0158-sup0037-02-Christiana-Care-HS-vol41.pdf.
62. Mann, H., Clinical Trial Protocols: Agreements Between the FDA and Industrial Sponsors. The Lancet, 2002. 360: p. 13451346.

Sameer Apte (B.Sc. 2007) is a graduate of McGill University's joint majors program in Physiology and Physics. Throughout his undergraduate studies, Sameer cultivated a scientific interest in tissue engineering in relation to the cardiovascular system. In addition, Sameer is peripherally interested in the bioethics of clinical medicine. In the future, Sameer wishes to pursue a medical career which synthesizes clinical practice and academic investigation. 